\title{
Symptomatic multifocal avascular necrosis in an adolescent with neuropsychiatric systemic lupus erythematosus
}

\author{
Rabia Miray Kisla Ekinci ${ }^{1}$, Sibel Balcı ${ }^{1}$, Gonca Celik ${ }^{2}$, Dilek Dogruel ${ }^{3}$, Derya Ufuk Altıntas ${ }^{3}$, \\ Mustafa Yilmaz ${ }^{1}$ \\ ${ }^{1}$ Department of Pediatric Rheumatology, Cukurova University Faculty of Medicine, Adana, Turkey \\ ${ }^{2}$ Department of Child and Adolescent Psychiatry, Cukurova University Faculty of Medicine, Adana, Turkey \\ ${ }^{3}$ Department of Pediatric Allergy and Immunology, Cukurova University Faculty of Medicine, Adana, Turkey
}

\begin{abstract}
Systemic lupus erythematosus (SLE) is an autoimmune disorder characterized by malar rash, oral ulcers, arthralgia, photosensitivity and nephritis. Herein, we report a rare comorbidity, multiple avascular necrosis (AVN), in an adolescent SLE patient and also highlight the importance of risk factors for this comorbidity with a brief literature review.

A 13-year-old female patient was admitted with severe headache, visual plus auditory hallucinations, polyarthritis and a history of recurrent oral ulcers. Acneiform malar rash, arthritis, cytopenia, low complement levels and autoantibody positivity yielded SLE diagnosis. We diagnosed her as having multifocal AVN after the 4th dose of cyclophosphamide, with bilateral knee pain and swelling and typical geographical lesions on magnetic resonance imaging.

Avascular necrosis is a rare comorbidity of SLE and neuropsychiatric involvement, cyclophosphamide administration and severe disease may be the possible risk factors in addition to corticosteroid use. Further multicenter studies investigating the possible risk factors of AVN with a large number of patients are needed.
\end{abstract}

Key words: avascular necrosis of bone, adolescent, osteonecrosis, systemic lupus erythematosus.

\section{Introduction}

Systemic lupus erythematosus (SLE) is a multisystemic autoimmune disorder in which local deposition of anti-nuclear antibodies and activation of the complement system take part. The most common manifestations are malar rash, oral aphthous ulcers, arthralgia, photosensitivity and nephritis. Pediatric cases account for $10-20 \%$ of all patients with SLE and have poorer prognosis with higher incidence of renal and central nervous system involvement [1].

Avascular necrosis (AVN), also known as osteonecrosis, is a disorder characterized by cessation of the blood supply and subsequent death of the bone marrow and trabecular bone. It is a well-known complication of SLE $[2,3]$. Avascular necrosis affects particularly femoral head, femoral condyles, proximal tibia and ankles. Mul- tifocal AVN is found in approximately $57-81 \%$ of patients with $\mathrm{AVN}$ and is characterized by the involvement of three or more separate anatomic sites [2, 4].

In view of the lack of information on prevalence and risk factors of AVN in juvenile SLE (jSLE), herein we describe a distinctive pediatric patient with neuropsychiatric (NP) SLE, who subsequently developed symptomatic multifocal AVN resembling polyarthritis.

\section{Case report}

A 13-year-old female patient was referred to Department of Pediatric Rheumatology with bilateral knee, wrist and hand pain and swelling for the last 3 months and recurrent oral aphthous ulcers for 5 years. The patient had suffered from severe headache and visual/ auditory hallucinations lasting two weeks before her

Address for correspondence:

Rabia Miray Kisla Ekinci, Department of Pediatric Rheumatology, Cukurova University Faculty of Medicine, Saricam, 01131 Adana, Turkey, e-mail: mir_kisla@hotmail.com

Submitted: 10.01.2019; Accepted: 28.05.2019 
joint complaints. Physical examination was normal except for the malar rash and arthritis in wrists and ankles.

Initial laboratory results revealed normal urine analysis, leukopenia, slight thrombocytopenia, mildly elevated liver enzymes and markedly reduced serum complement levels. Antinuclear antibody (ANA), anti-double-stranded DNA (dsDNA) and anti-SSA antibodies were positive, whereas antibodies for other extractable nuclear antigens (ENA) were negative. Autoimmune hepatitis spectrum antibody as smooth muscle antibody (SMA) was positive, whereas liver-kidney-muscle-1 antibody (anti-LKM1) was negative. We diagnosed the patient as having SLE and autoimmune hepatitis $(\mathrm{AlH})$ and initiated treatment with methylprednisolone, in a maximum oral dose of $48 \mathrm{mg} /$ day, hydroxychloroquine and azathioprine.

For the next 4 months, arthritis and skin rash were alleviated, but NP SLE symptoms increased. Central nervous system imaging with magnetic resonance (MR) and angiography did not reveal any organic abnormalities. Nonetheless we ceased azathioprine and initiated risperidone and monthly intravenous cyclophosphamide.
Neuropsychiatric symptoms improved and serum complement levels returned to normal; thus we tapered the methylprednisolone dose to $8 \mathrm{mg} /$ day. Thereafter, the patient began to suffer from bilateral knee pain and swelling. At the $4^{\text {th }}$ dose of cyclophosphamide and after a $5.3 \mathrm{~g}(94.7 \mathrm{mg} / \mathrm{kg})$ cumulative dose of methylprednisolone, a diagnosis of symptomatic multifocal AVN was made by detecting typical geographical lesions on MR imaging although classical radiography was normal (Fig. 1).

Further laboratory investigations were all normal except for the presence of thalassemia trait. Bone densitometry revealed a total vertebral $Z$ score of -2.9 . One month later, the patient clinically improved with ibuprofen and physical exercise in addition to dietary vitamin D and calcium supplementation. Laboratory results are summarized in Table I. The consent for the presentation of this case was obtained from both parents and the patient.

\section{Discussion}

The pathogenetic mechanisms of AVN are still not exactly understood, but vascular injury, hypercoagula-
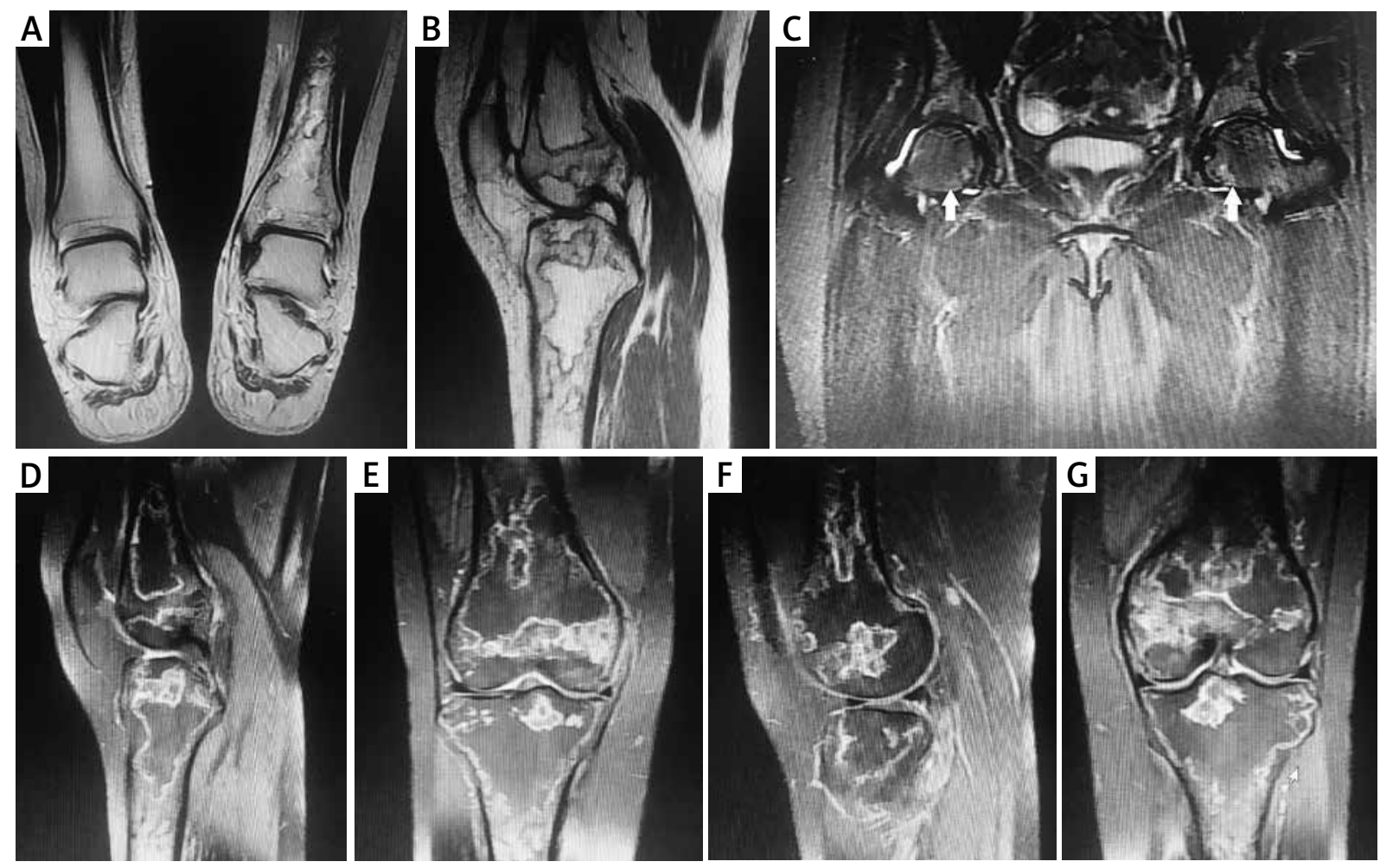

Fig. 1. Characteristic lesions of avascular necrosis on magnetic resonance imaging. Avascular necrosis with geographic-appearing lesions on coronal T1 image of the right distal tibia (A) and sagittal T1-weighted images of the right knee (proximal tibia and distal femur) (B). Coronal T2 magnetic resonance image shows bilateral hyperintensity at the bottom of femoral head (arrows) revealing medullary infarct (C). T2-weighted fat suppressed images yield geographic appearance compatible with avascular necrosis right proximal tibia and distal femur on sagittal (D) and coronal view (E), left proximal tibia and distal femur on sagittal (F) and coronal view $(\mathrm{G})$ respectively. 
Table I. Laboratory parameters of the patient with juvenile systemic lupus erythematosus at diagnosis and occurrence of multifocal avascular necrosis

\begin{tabular}{|c|c|c|c|}
\hline Parameters & First administration & At AVN diagnosis & Normal values \\
\hline Hemoglobin (g/dl) & 11.5 & 11.3 & $12-15.5$ \\
\hline Leukocytes $\left(/ \mathrm{mm}^{3}\right)$ & 3,120 & 6,100 & $4,000-11,500$ \\
\hline Thrombocytes $\left(/ \mathrm{mm}^{3}\right)$ & 143,000 & 240,000 & $150,000-450,000$ \\
\hline $\operatorname{ESR}(\mathrm{mm} / \mathrm{h})$ & 30 & 21 & $0-15$ \\
\hline CRP $(\mathrm{mg} / \mathrm{dl})$ & 0.4 & 0.71 & $0-0.5$ \\
\hline SGOT (U/ml) & 197 & 15 & $5-40$ \\
\hline SGPT $(\mathrm{U} / \mathrm{ml})$ & 111 & 11 & $5-40$ \\
\hline C3 (mg/dl) & 17.4 & 113 & 88-201 \\
\hline $\mathrm{C} 4$ (mg/dl) & 4.3 & 16.3 & $14.9-22.1$ \\
\hline ANA (IFA) & pos & neg & $<1 / 100$ titer \\
\hline Anti-dsDNA (IU/ml) & 193 & 20.4 & $0-15$ \\
\hline Anti-Sm (U/ml) & 4.9 & 0.34 & $0-15$ \\
\hline Anti-RNP & 5.7 & 0.35 & $0-15$ \\
\hline Anti-SSA & 33 & 1.38 & $0-15$ \\
\hline Anti-SSB & 10 & 1.74 & $0-15$ \\
\hline AMA $(\mathrm{U} / \mathrm{ml})$ & 4.8 & ND & $0-5$ \\
\hline Anti-LKM1 (IFA) & neg & ND & $<1 / 10$ titer \\
\hline SMA (IFA) & pos & ND & $<1 / 40$ titer \\
\hline Anticardiolipin lgG (U/ml) & 8.9 & 2 & $0-10$ \\
\hline Anticardiolipin lgM (U/ml) & 5.3 & 1.8 & $0-7$ \\
\hline Anti- $\beta 2$ glycoprotein 1 (U/ml) & 0.2 & neg & $0-5$ \\
\hline Lupus anticoagulant & ND & neg & ND \\
\hline Total triglyceride (mg/dl) & ND & 90.8 & $50-150$ \\
\hline LDL $(\mathrm{mg} / \mathrm{dl})$ & ND & 102 & $80-150$ \\
\hline $\mathrm{HDL}(\mathrm{mg} / \mathrm{dl})$ & ND & 55 & $35-60$ \\
\hline Total cholesterol (mg/dl) & ND & 175 & $150-200$ \\
\hline $\mathrm{Hb}$ electrophoresis (\%) (HbA/HbA2) & ND & $96.1 / 3.9$ & $95-98 / 1.5-3.5$ \\
\hline Serum calcium (mg/dl) & 12 & 8.9 & $8.8-10.3$ \\
\hline Serum phosphorus (mg/dl) & 3.4 & 3.7 & $2.4-4.7$ \\
\hline Alkaline phosphatase (IU/I) & 126 & 54 & $67-372$ \\
\hline Serum vitamin $D(\mathrm{ng} / \mathrm{ml})$ & ND & 18.3 & $20-60$ \\
\hline
\end{tabular}

AVN - avascular necrosis, ESR - erythrocyte sedimentation rate, CRP-C-reactive protein, SGOT - serum glutamic oxaloacetic transaminase, SGPT - serum glutamic pyruvic transaminase, C3-complement 3, C4-complement 4, ANA - antinuclear antibody, anti-DNA - anti-double stranded DNA antibody, anti-Sm - anti-Smith, anti-RNP - anti-ribonucleoprotein, anti-SSA - anti-Sjögren's-syndrome-related antigen A, anti-SSB - anti-Sjögren's-syndrome-related antigen B, AMA - antimitochondrial antibody, anti-LKM1 - anti-liver kidney muscle 1, SMAsmooth muscle antibody, LDL - low-density lipoprotein, HDL - high-density lipoprotein, $\mathrm{Hb}$ - hemoglobin, ND - not determined.

bility, fat embolism and inhibition of angiogenesis have been suggested [2]. Although corticosteroid treatment is thought to be the major independent risk factor in SLE patients, AVN has also been reported even in the absence of steroid use, as an early musculoskeletal manifestation in SLE [3-6].

Another prospective study showed that patients with antiphospholipid syndrome (APS) without previous steroid treatment were also predisposed to AVN. This situation raised the question whether APS is a major risk factor for AVN development [7].

Additionally, CNS involvement, nephritis, vitamin D deficiency, hypertriglyceridemia, hypertension, and immunosuppressive drugs have been suggested to influence AVN development in SLE $[2,4,8]$. Previously, antiphospholipid antibodies (APLs) were linked with AVN, 


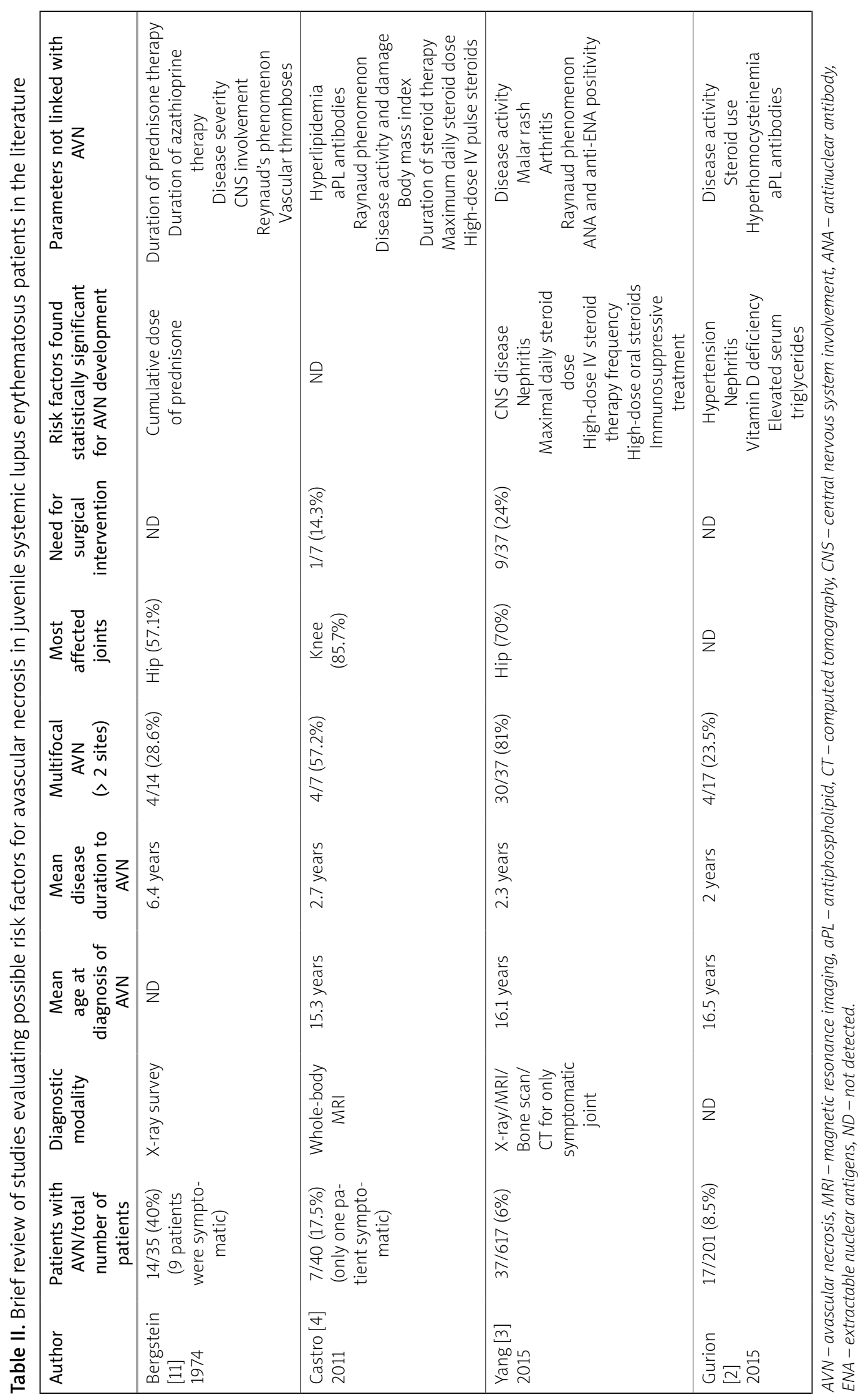


but recent studies disproved that [2, 4-8]. Also, in a recent meta-analysis, arthritis, cushingoid appearance, gastrointestinal involvement, hypertension, oral ulcers, nephritis, vasculitis, cytotoxic drugs, and steroid treatment with both cumulative and daily dose were found to be related to AVN development. In contrast, APLS, CNS involvement and Raynaud's phenomenon are not associated with AVN [9].

Diagnosis of AVN is based on imaging. Plain radiographs may show late changes including mottling of the bone trabecular pattern, depression or fragmentation, joint space narrowing and irregular sclerosis. MRI is more sensitive in the early phase of AVN and the most demonstrative finding is geographic areas of low and high signal intensities, whereas bone marrow edema, subchondral collapse and effusion may also be seen $[2,4,5]$. Our patient had normal X-ray but typical MRI findings. Therefore we suggest that AVN should be considered in jSLE patient with arthralgia and normal X-ray and it is advisable to perform MR examination in such cases.

The frequency of AVN in SLE (6\%) was encountered in only one multicenter adult study from our country [10]. We do not know the exact AVN rate in juvenile SLE patients; thus the possible risk factors were not examined in the pediatric population in our country.

To date, only 4 relevant studies have investigated AVN frequency and risk factors in solely juvenile SLE patients. In the preliminary study including 14 jSLE with AVN diagnosed with X-ray, cumulative steroid dose was linked with AVN, but duration of steroid and azathioprine therapy, disease severity, CNS involvement, Raynaud's phenomenon and vascular thromboses had no effect on AVN development [11].

Castro et al. [4] prospectively performed whole body MRI in 40 juvenile SLE patients and suggested that steroid treatment did not influence AVN in jSLE. The small sample size was the major limitation of this study. Two recent jSLE cohorts eliminated this limitation and showed AVN frequency of $6 \%$ and $8.5 \%$ in 617 and 201 patients respectively.

Yang et al. [3] reported a higher rate of CNS disease, nephritis, maximal daily prednisolone dose, high-dose i.v. methylprednisolone therapy frequency, high-dose (oral) corticosteroids and immunosuppressive drug administration in the AVN group than without. Disease activity, malar rash, arthritis, Raynaud phenomenon, ANA and anti-ENA positivity were similar between these groups [3]. Similarly, nephritis was linked to AVN in the other study. Besides hypertension, vitamin D deficiency and elevated serum triglycerides were higher in the AVN group. Interestingly, corticosteroid use was similar between patients with and without AVN [2]. Studies investigating possible risk factors for AVN in jSLE patients are summarized in Table II.

In regard to aforementioned conflicting results in the literature, further studies are needed to define the risk factors for AVN in jSLE, which may lead to clarifying the pathogenesis and preventing AVN in pediatric cases.

Our patient had several risk factors for AVN including oral ulcers, arthritis, steroid and cytotoxic treatment and CNS involvement. Since CNS involvement, requirement for cytotoxic treatment and higher cumulative steroid dose can be linked to a severe disease phenotype, higher inflammation may lead to AVN development.

By contrast, no associations were found between oral aphthous and AVN in SLE patients in a recent meta-analysis [12]. Similarly, a recent comprehensive study, including 1219 adults with SLE, also concluded that high-dose corticosteroid and immunosuppressant use are the principal risk factors for developing AVN [13].

Hemoglobin concentration and electrophoresis in our patient revealed $\beta$-thalassemia trait which was not previously linked to AVN. Nonetheless, we would expect that thalassemia trait may be protective against AVN due to mild anemia, contrary to our case. Further multicenter case-control studies are needed to confirm the influence of thalassemia trait on AVN development in jSLE patients from the Mediterranean region.

In view of the heterogeneity of the results of different studies and even the aforementioned meta-analysis, there is no consensus about the exact pathogenesis of AVN in SLE and even more so in the juvenile form of the disease.

\section{Conclusions}

The described case of a young female patient with jSLE treated with steroids and cyclophosphamide points to frequent AVN risk factors, but may also be a contribution to a wider discussion of the risk of AVN in such patients. AVN should be considered in differential diagnosis of arthritis in patients with SLE and jSLE particularly in the case of prolonged use of steroids and cyclophosphamide administration.

The authors declare no conflicts of interest.

\section{References}

1. Malattia C, Martini A. Paediatric-onset systemic lupus erythematosus. Best Pract Res Clin Rheumatol 2013; 27: 351-362.

2. Gurion R, Tangpricha V, Yow E, et al. Avascular necrosis in pediatric systemic lupus erythematosus: a brief report and review of the literature. Pediatr Rheumatol Online J 2015; 3: 13.

3. Yang Y, Kumar S, Lim LS, et al. Risk Factors for Symptomatic Avascular Necrosis in Childhood-onset Systemic Lupus Erythematosus. J Rheumatol 2015; 42: 2304-2309. 
4. Castro TC, Lederman H, Terreri MT, et al. The use of joint-specific and whole-body MRI in osteonecrosis: a study in patients with juvenile systemic lupus erythematosus. Br J Radiol 2011; 84: 621-628.

5. Gladman DD, Dhillon N, Su J, et al. Osteonecrosis in SLE: prevalence, patterns, outcomes and predictors. Lupus 2018; 27 : 76-81.

6. Adikari M, Gunawardane A, Illangantilaka S, et al. A case of systemic lupus erythematosus presenting as bilateral avascular necrosis of femur. BMC Res Notes 2016; 9: 392.

7. Tektonidou MG, Malagari K, Vlachoyiannopoulos PG, et al. Asymptomatic avascular necrosis in patients with primary antiphospholipid syndrome in the absence of corticosteroid use: a prospective study by magnetic resonance imaging. Arthritis Rheum 2003; 48: 732-736.

8. Mok CC, Lau CS, Wong RW. Risk factors for avascular bone necrosis in systemic lupus erythematosus. Br J Rheumatol 1998; 37: $895-900$
9. Zhu KK, Xu WD, Pan HF, et al. The risk factors of avascular necrosis in patients with systemic lupus erythematosus: a metaanalysis. Inflammation 2014; 37: 1852-1864.

10. Sayarlioglu M, Yuzbasioglu N, Inanc M, et al. Risk factors for avascular bone necrosis in patients with systemic lupus erythematosus. Rheumatol Int 2012; 32: 177-182.

11. Bergstein JM, Wiens C, Fish A, et al. Avascular necrosis of bone in systemic lupus erythematosus. J Pediatr 1974; 85: 31-35.

12. Nevskaya T, Gamble MP, Pope JE. A meta-analysis of avascular necrosis in systemic lupus erythematosus: prevalence and risk factors. Clin Exp Rheumatol 2017; 35: 700-710.

13. Kwon HH, Bang SY, Won S, et al. Synergistic effect of cumulative corticosteroid dose and immunosuppressants on avascular necrosis in patients with systemic lupus erythematosus. Lupus 2018; 27: 1644-1651. 